\title{
Article \\ Applications of Inequalities in the Complex Plane Associated with Confluent Hypergeometric Function
}

\author{
Georgia Irina Oros
}

check for updates

Citation: Oros, G.I. Applications of Inequalities in the Complex Plane Associated with Confluent Hypergeometric Function. Symmetry 2021, 13, 259. https://doi.org/ 10.3390/sym13020259

Academic Editor: Alina Alb Lupas Received: 14 January 2021 Accepted: 1 February 2021 Published: 4 February 2021

Publisher's Note: MDPI stays neutral with regard to jurisdictional clai$\mathrm{ms}$ in published maps and institutional affiliations.

Copyright: () 2021 by the author. Licensee MDPI, Basel, Switzerland. This article is an open access article distributed under the terms and conditions of the Creative Commons Attribution (CC BY) license (https:// creativecommons.org/licenses/by/ $4.0 /)$.
Department of Mathematics and Computer Science, Faculty of Informatics and Sciences, University of Oradea, 410087 Oradea, Romania; georgia_oros_ro@yahoo.co.uk

\begin{abstract}
The idea of inequality has been extended from the real plane to the complex plane through the notion of subordination introduced by Professors Miller and Mocanu in two papers published in 1978 and 1981. With this notion came a whole new theory called the theory of differential subordination or admissible functions theory. Later, in 2003, a particular form of inequality in the complex plane was also defined by them as dual notion for subordination, the notion of differential superordination and with it, the theory of differential superordination appeared. In this paper, the theory of differential superordination is applied to confluent hypergeometric function. Hypergeometric functions are intensely studied nowadays, the interest on the applications of those functions in complex analysis being renewed by their use in the proof of Bieberbach's conjecture given by de Branges in 1985. Using the theory of differential superodination, best subordinants of certain differential superordinations involving confluent (Kummer) hypergeometric function are stated in the theorems and relation with previously obtained results are highlighted in corollaries using particular functions and in a sandwich-type theorem. An example is also enclosed in order to show how the theoretical findings can be applied.
\end{abstract}

Keywords: differential superordination; analytic function; convex function; univalent function; subordinant; best subordinant; confluent hypergeometric function

\section{Introduction}

In the case of real-valued functions, bounds on a function $f$ are often determined from an inequality involving several derivatives of the function. As a simple example, a function $f$ twice continuously differentiable on an interval $I=(-1,1)$ can be considered and suppose that the differential operator

$$
D[f](t)=t^{2} f^{\prime \prime}(t)+4 t f^{\prime}(t)+2 f(t)+6 t=\left[t^{2} f(t)+t^{3}\right]^{\prime \prime}
$$

satisfies

$$
0<D[f](t)<2
$$

for $t \in I$. It is easily seen that such a function has the property that $-1<f(t)<2$ for $t \in I$. That can be written as

$$
D[f](t) \subset(0,2) \Rightarrow f(I) \subset(-1,2) .
$$

In two articles in 1978 [1] and 1981 [2] the authors extended these ideas involving differential inequalities for the real-valued functions to complex-valued functions setting the basis for a new theory later named theory of differential subordinations or admissible functions theory which has developed rapidly in the following years since it provides means for proving a lot easier known results and for easily obtaining interesting, original ones.

The idea of introducing the notion of subordination originated in the observation that the differential inequality of the form (1) does not have a direct analog for complex-valued 
functions since the real-valued function $f(t)$ cannot be replaced by a complex-valued function $f(z)$, but the first inclusion from (2) has a natural complex analog in the form

$$
D[f](U) \subset \Omega
$$

where

$$
D[f](z)=t^{2} f^{\prime \prime}(z)+4 z f^{\prime}(z)+2 f(z)+6 z,
$$

$U$ denoting the unit disc of the complex plane and $\Omega \subset \mathbb{C}$.

If $f: U \rightarrow \mathbb{C}$ satisfies this inclusion then, following the implication in relation (2), the question is whether there exists a "smallest" set $\Delta \subset \mathbb{C}$ such that

$$
D[f](U) \subset \Omega \Rightarrow f(U) \subset \Delta
$$

for the differential subordination theory, and a "smallest" set $\Omega$ for which

$$
\Omega \subset D[f](U) \Rightarrow \Delta \subset f(U)
$$

for the differential superordination theory, introduced in [3].

The results presented in the original part of this paper will answer this last question. Throughout the paper, we use the well-known classes:

- $H(U)$ denotes the class of analytic functions in the unit disc of the complex plane;

- $\quad$ For $n$ a positive integer and $a \in \mathbb{C}$, let

$$
H[a, n]=\left\{f \in H(U): f(z)=a+a_{n} z^{n}+a_{n+1} z^{n+1}+\ldots, z \in U\right\}, \text { with } H_{0}=H[0,1] ;
$$

- $A_{n}=\left\{f \in H(U): f(z)=z+a_{n+1} z^{n+1}+\ldots, z \in U\right\}$, with $A_{1}=A$;

- $K=\left\{f \in H(U): \operatorname{Re} \frac{z f^{\prime \prime}(z)}{f^{\prime}(z)}+1>0, z \in U\right\}$ denotes the class of convex functions in the unit disc;

- $S=\left\{f \in H(U): f(z)=z+a_{2} z^{2}+a_{3} z^{3}+\ldots, f\right.$ univalent in $\left.U\right\}$.

The theory of differential superordination is based on the following definitions:

Definition 1. ([3]) Let $f$ and $F$ be members of $H(U)$. The function $f$ is said to be subordinate to $F$, or $F$ is said to be superordinate to $f$, if there exists a function $w$, analytic in $U$, with $w(0)=0$ and $|w(z)|<1$ and such that $f(z)=F(w(z))$. In such a case we write $f \prec F$ or $f(z) \prec F(z)$. If $F$ is univalent, then $f \prec F$ if and only if $f(0)=F(0)$ and $f(U) \subset F(U)$.

Definition 2. ([3]) Let $\varphi(r, s, t ; z): \mathbb{C}^{3} \times \bar{U} \rightarrow \mathbb{C}$ and let $h$ be analytic in $U$. If $p$ and $\varphi\left(p(z), z p^{\prime}(z), z^{2} p^{\prime \prime}(z) ; z\right)$ are univalent in $U$ and satisfy the (second-order) differential superordination

$$
h(z) \prec \varphi\left(p(z), z p^{\prime}(z), z^{2} p^{\prime \prime}(z) ; z\right)
$$

then $p$ is called a solution of the differential superordination. An analytic function $q$ is called a subordinant of the solutions of the differential superordination or more simply a subordinant, if $q \prec p$ for all $p$ satisfying (5). A subordinant $\widetilde{q}$ that satisfies $q \prec \widetilde{q}$ for all subordinants $q$ of (5) is said to be the best subordinant of (5). Note that the best subordinant is unique up to a rotation of $U$.

Definition 3. ([3]) We denote by $Q$ the set of functions $f$ that are analytic and injective on $\bar{U} \backslash E(f)$ where

$$
E(f)=\left\{\zeta \in \partial U: \lim _{z \rightarrow \zeta} f(z)=\infty\right\}
$$

and are such that $f^{\prime}(\zeta) \neq 0$, for $\zeta \in \partial U \backslash E(f)$. The subclass of $Q$ for which $f(0)=a$ is denoted by $Q(a)$. 
Another notion used for obtaining the original results is that of subordination chains as it can be found in [4]:

Definition 4. ([4]) A function $L(z, t), z \in U, t \geq 0$ is a subordination chain if $L(\cdot, t)$ is analytic and univalent in $U$ for all $t \geq 0, L(z, \cdot)$ is contiunously differentiable on $\mathbb{R}^{+}$for all $z \in U$ and $L(z, s) \prec L(z, t)$ when $0 \leq s \leq t$.

The applications of the hypergeometric functions in the field of complex analysis became obvious and worthy of investigation after L. de Branges used them in proving the famous Bieberbach's Conjecture [5]. One of the first papers to study the implications of using hypergeometric functions in the theory of univalent functions is the paper published by Miller and Mocanu in 1990 [6]. In their paper, Gaussian and confluent (Kummer) hypergeometric functions are studied and conditions for their univalence are stated which inspired further study.

The confluent (Kummer) hypergeometric function of the first kind has the following definition:

Definition 5. ([7], p. 5) Let $a$ and $c$ be complex numbers with $c \neq 0,-1,-2, \ldots$ and consider

$$
\phi(a, c ; z)={ }_{1} F_{1}(a, c ; z)=1+\frac{a}{c} \cdot \frac{z}{1 !}+\frac{a(a+1)}{c(c+1)} \cdot \frac{z^{2}}{2 !}+\ldots, z \in U .
$$

This function is called confluent (Kummer) hypergeometric function, is analytic in $\mathbb{C}$ and satisfies Kummer's differential equation:

$$
z \cdot w^{\prime \prime}(z)+[c-z] \cdot w^{\prime}(z)-a \cdot w(z)=0
$$

If we let

$$
(d)_{k}=\frac{\Gamma(d+k)}{\Gamma(d)}=d(d+1)(d+2) \ldots(d+k-1) \text { and }(d)_{0}=1
$$

then (4) can be written in the form

$$
\phi(a, c ; z)=\sum_{k=0}^{\infty} \frac{(a)_{k}}{(c)_{k}} \cdot \frac{z^{k}}{k !}=\frac{\Gamma(c)}{\Gamma(a)} \cdot \sum_{k=0}^{\infty} \frac{\Gamma(a+k)}{\Gamma(c+k)} \cdot \frac{z^{k}}{k !} .
$$

In paper [6] the authors have determined conditions on $a, c \in \mathbb{R}, c \neq 0,-1,-2, \ldots$ for the function $\phi$ to be univalent in $U$. In paper [8], the theory of differential subordinations was used in order to obtain new conditions for the univalence of this function considering $a, c \in \mathbb{C}, c \neq 0,-1,-2, \ldots$.

Not much study was done using differential superordination theory on confluent hypergeometric function. Some superordination results were obtained for the generalized hypergeometric function as it can be seen in papers [9-11] some superordination results were stated in the form of sandwich-type theorems.

Considering the results obtained in paper [8] and motivated by the interesting outcomes published implementing the theory of differential superordination, means of this theory are used in this paper in order to investigate confluent (Kummer) hypergeometric function taking $a, c \in \mathbb{C}, c \neq 0,-1,-2, \ldots$. The results obtained here are connected to those from paper [8] through some corollaries and a sandwich-type theorem.

A lemma is required in order to prove the original results contained in the next section.

Lemma 1. ([7]) Let $p \in Q(a)$ and let $q(z)=a+a_{n} z^{n}+a_{n+1} z^{n+1}+\ldots$ be analytic in $U$ with $q(z) \not \equiv(a)$ and $n \geq 1$. If $q$ is not subordinate to $p$, then there exists points $z_{0}=r_{0} e^{i \theta_{0}} \in U$ and $\zeta_{0} \in \partial U \backslash E(p)$ and an $m \geq n \geq 1$ for which $q\left(U_{r_{0}}\right) \subset p(U)$ and

(i) $q\left(z_{0}\right)=p\left(\zeta_{0}\right)$; 
(ii) $z_{0} q^{\prime}\left(z_{0}\right)=m \zeta_{0} p^{\prime}\left(\zeta_{0}\right)$ and

(iii) $\operatorname{Re} \frac{z_{0} q^{\prime \prime}\left(z_{0}\right)}{q^{\prime}\left(z_{0}\right)}+1 \geq m \operatorname{Re}\left[\frac{\zeta_{0} p^{\prime \prime}\left(\zeta_{0}\right)}{p^{\prime}\left(\zeta_{0}\right)}+1\right]$.

\section{Results}

The original results contained in this paragraph answer the question generated by the applications of the inequalities in the complex plane written in the form of relation (4).

Among the novelties that the results presented in this paper bring is the use of the theory of differential superordination in order to study the confluent hypergeometric function and obtain interesting superordinations for which it is the best subordinant. Another aspect of novelty is the geometrical interpretation of the obtained superodinations in terms of set inclusions which give sufficient conditions for the confluent hypergeometric function to be a function with positive real part.

The first superordination result is contained in the following theorem. Its proof is very simple since Lemma 1 presented in the Introduction is applied, also known as Miller-Mocanu lemma, a strong result which has been used in obtaining many interesting outcomes both in the theory of differential subordination and in the theory of differential superordination. Theorem 1 is very important as it facilitates the proof of the original results in the next theorems.

Theorem 1. Let $\phi(a, c ; z)=1+\frac{a}{c} \cdot \frac{z}{1 !}+\frac{a(a+1)}{c(c+1)} \cdot \frac{z^{2}}{2 !}+\ldots, a, c \in \mathbb{C}, c \neq 0,-1,-2, \ldots$, defined by (6), $\varphi: \mathbb{C}^{3} \times \bar{U} \rightarrow \mathbb{C}$ and $h$ be analytic in $U$. Suppose that the differential equation

$$
\varphi\left(\phi(a, c ; z), z \phi^{\prime}(a, c ; z), z^{2} \phi^{\prime \prime}(a, c ; z) ; z\right)=h(z)
$$

has a solution $q(z)=\phi(a, c ; z) \in Q(1)$.

If $p \in Q(1)$ and $\varphi\left(p(z), z p^{\prime}(z), z^{2} p^{\prime \prime}(z) ; z\right)$ is univalent in $U$, then

$$
h(z) \prec \varphi\left(p(z), z p^{\prime}(z), z^{2} p^{\prime \prime}(z) ; z\right)
$$

implies

$$
q(z)=\phi(a, c ; z) \prec p(z), z \in U
$$

and $q(z)=\phi(a, c ; z)$ is the best subordinant.

Proof. Using Definition 1, from (9) we have

$$
h(U) \subset \varphi(U)
$$

since $\varphi\left(p(z), z p^{\prime}(z), z^{2} p^{\prime \prime}(z) ; z\right)$ is univalent in $U$.

Suppose that functions $p, q, h$ satisfy the conditions from Lemma 1 , in $\bar{U}$.

Assume $q(z)=\phi(a, c ; z) \nprec p(z)$. By Lemma 1 there exists points $z_{0}=r_{0} e^{i \theta_{0}} \in U$ and $\zeta_{0} \in \partial U$ and $m \geq n \geq 1$ that satisfy the conditions:

$$
\begin{gathered}
\phi\left(a, c ; z_{0}\right)=p\left(\zeta_{0}\right), \\
z_{0} \phi^{\prime}\left(a, c ; z_{0}\right)=m \zeta_{0} p^{\prime}\left(\zeta_{0}\right), \\
z_{0}^{2} \phi^{\prime \prime}\left(a, c ; z_{0}\right)=\zeta_{0}^{2} p^{\prime \prime}\left(\zeta_{0}\right) . \\
\text { From } r=\phi\left(a, c ; z_{0}\right)=p\left(\zeta_{0}\right), s=\frac{z \phi^{\prime}\left(a, c ; z_{0}\right)}{m}=\zeta_{0} p^{\prime}\left(\zeta_{0}\right), t=\zeta_{0}^{2} p^{\prime \prime}\left(\zeta_{0}\right), \text { we have } \\
\varphi\left(p\left(\zeta_{0}\right), \zeta_{0} p^{\prime}\left(\zeta_{0}\right), \zeta_{0}^{2} p^{\prime \prime}\left(\zeta_{0}\right)\right)=\varphi\left(\phi\left(a, c ; z_{0}\right), z_{0} \phi^{\prime}\left(a, c ; z_{0}\right), z_{0}^{2} \phi^{\prime \prime}\left(a, c ; z_{0}\right)\right) \in h(U) .
\end{gathered}
$$


Since this contradicts (10) we must have

$$
\phi(a, c ; z) \prec p(z) \text { or } \phi(U) \subset p(U), z \in U .
$$

Since $q(z)=\phi(a, c ; z)$ satisfies the Equation (8), the conclusion is that $q$ is the best subordinant.

Remark 1. From this theorem we see that the problem of finding the best subordinant of (9) essentially reduces to showing that the differential Equation (8) has a univalent solution.

The conclusion of the theorem can be written in the symmetric form

$$
\varphi\left(q(z), z q^{\prime}(z), z^{2} q^{\prime \prime}(z) ; z\right) \prec \varphi\left(p(z), z p^{\prime}(z), z^{2} p^{\prime \prime}(z) ; z\right)
$$

or

$$
\left\{\varphi\left(q(z), z q^{\prime}(z), z^{2} q^{\prime \prime}(z) ; z\right)\right\} \subset\left\{\varphi\left(p(z), z p^{\prime}(z), z^{2} p^{\prime \prime}(z) ; z\right)\right\}
$$

which implies

$$
q(z) \prec p(z), \quad \text { or } \quad q(U) \subset p(U), z \in U .
$$

We can simplify Theorem 1 for the case of first order differential superordination. The simplified form contained in Theorem 2 is used in the proof of the next theorems, hence it is also a key result of this paper, next to Theorem 1.

Theorem 2. Let $\phi(a, c ; z)=1+\frac{a}{c} \cdot \frac{z}{1 !}+\frac{a(a+1)}{c(c+1)} \cdot \frac{z^{2}}{2}+\ldots, a, c \in \mathbb{C}, c \neq 0,-1,-2, \ldots$, defined by (6), $\varphi: \mathbb{C}^{2} \times \bar{U} \rightarrow \mathbb{C}$ and $h$ be analytic in $U$.

Suppose that

$$
\varphi\left(\phi(a, c ; z), t z \phi^{\prime}(a, c ; z)\right) \in h(U) \text { for } z \in U \text { and } 0<t \leq \frac{1}{n} \leq 1 .
$$

If $p \in Q(1)$ and $\varphi\left(p(z), z p^{\prime}(z) ; z\right)$ is univalent in $U$, then

$$
h(z)=\varphi\left(\phi(a, c ; z), z \phi^{\prime}(a, c ; z) ; z\right) \prec \varphi\left(p(z), z p^{\prime}(z) ; z\right) \quad \text { or } \quad h(U) \subset \varphi(U)
$$

implies

$$
q(z)=\phi(a, c ; z) \prec p(z), \quad \text { or } \quad \phi(U) \subset p(U), z \in U
$$

and $q(z)=\phi(a, c ; z)$ is the best subordinant.

The first result which appeals to Theorem 1 for its proof is the next theorem which also generates two interesting corollaries for particular uses of function $p$ involved giving sufficient conditions for the confluent hypergeometric function to be a function with positive real part.

Theorem 3. Let $q(z)=\phi(a, c ; z)=1+\frac{a}{c} \cdot \frac{z}{1 !}+\frac{a(a+1)}{c(c+1)} \cdot \frac{z^{2}}{2 !}+\ldots, a, c \in \mathbb{C}, c \neq 0,-1,-2, \ldots$, defined by (6) and let $h$ be defined by

$$
h(z)=\phi(a, c ; z)+z \phi^{\prime}(a, c ; z)+z^{2} \phi^{\prime \prime}(a, c ; z) .
$$

If $p \in \mathcal{H}[1,1] \cap Q, p(z)+z p^{\prime}(z)+z^{2} p^{\prime \prime}(z)$ is univalent in $U$, and

$$
\phi(a, c ; z)+z \phi^{\prime}(a, c ; z)+z^{2} \phi^{\prime \prime}(a, c ; z) \prec p(z)+z p^{\prime}(z)+z^{2} p^{\prime \prime}(z)
$$

then

$$
\phi(a, c ; z) \prec p(z), z \in U \quad \text { or } \quad \phi(U) \subset p(U)
$$


and $q(z)=\phi(a, c ; z)$ is the best subordinant.

Proof. Let $\varphi: \mathbb{C}^{3} \times \bar{U} \rightarrow \mathbb{C}$, where

$$
\varphi(r, s, t)=r+s+t .
$$

For $r=\phi(a, c ; z), s=z \phi^{\prime}(a, c ; z), t=z^{2} \phi^{\prime \prime}(a, c ; z)$, we have

$$
\begin{gathered}
\varphi\left(\phi(a, c ; z), z \phi^{\prime}(a, c ; z), z^{2} \phi^{\prime \prime}(a, c ; z)\right) \\
=\phi(a, c ; z)+z \phi^{\prime}(a, c ; z)+z^{2} \phi^{\prime \prime}(a, c ; z)=h(z), z \in U .
\end{gathered}
$$

For $r=p(z), s=z p^{\prime}(z), t=z^{2} p^{\prime \prime}(z)$, we get

$$
\varphi\left(p(z), z p^{\prime}(z), z^{2} p^{\prime \prime}(z) ; z\right)=p(z)+z p^{\prime}(z)+z^{2} p^{\prime \prime}(z), z \in U .
$$

Using (18) and (19), superordination (16) becomes

$$
\varphi\left(\phi(a, c ; z), z \phi^{\prime}(a, c ; z), z^{2} \phi^{\prime}(a, c ; z) ; z\right) \prec \varphi\left(p(z), z p^{\prime}(z), z^{2} p^{\prime \prime}(z) ; z\right) .
$$

By using Theorem 1, we have

$$
q(z)=\phi(a, c ; z) \prec p(z), z \in U
$$

and $q(z)=\phi(a, c ; z)$ is the best subordinant.

Remark 2. Using $p(z)=\frac{1-z}{1+z}$, a convex function in $U$, from Theorem 3, we obtain the following corollary.

Corollary 1. Let $q(z)=\phi(a, c ; z)=1+\frac{a}{c} \cdot \frac{z}{1}+\frac{a(a+1)}{c(c+1)} \cdot \frac{z^{2}}{2 !}+\ldots, a, c \in \mathbb{C}, c \neq 0,-1,-2, \ldots$, defined by (6), let $\varphi: \mathbb{C}^{3} \times \bar{U} \rightarrow \mathbb{C}$ and let $h$ be defined by

$$
\begin{aligned}
& \qquad h(z)=\phi(a, c ; z)+z \phi^{\prime}(a, c ; z)+z^{2} \phi^{\prime \prime}(a, c ; z), z \in U . \\
& \text { If } p(z)=\frac{1-z}{1+z}, p(0)=1, \text { a convex function in } U \text {, and } \\
& p(z)+z p^{\prime}(z)+z^{2} p^{\prime \prime}(z)=\frac{-z^{3}+z^{2}+z+5}{(1+z)^{3}}
\end{aligned}
$$

is univalent in $U$ and satisfies

$$
\phi(a, c ; z)+z \phi^{\prime}(a, c ; z)+z^{2} \phi^{\prime \prime}(a, c ; z) \prec \frac{-z^{3}+z^{2}+z+5}{(1+z)^{3}}, z \in U
$$

or

$$
h(U) \subset \varphi(U),
$$

then

$$
\phi(a, c ; z) \prec \frac{1-z}{1+z}, z \in U, \quad \text { or } \quad \phi(U) \prec q(U),
$$

i.e.,

$$
\operatorname{Re} \phi(a, c ; z)>0, z \in U
$$

or

$$
\phi(U) \subset\{z \in \mathbb{C} \mid z=x+i y, x>0, y \in \mathbb{R}\}
$$

and $q(z)=\phi(a, c ; z)$ is the best subordinant. 
Proof. From relation (12) we have

$$
\phi(a, c ; z) \prec \frac{1-z}{1+z}, z \in U .
$$

Since $p(z)=\frac{1-z}{1+z}$ is a convex function in $U$, the differential superordination (22) is equivalent to

$$
\operatorname{Re} \phi(a, c ; z)>\operatorname{Re} \frac{1-z}{1+z}>0, z \in U
$$

or

$$
\phi(U) \subset\{z \in \mathbb{C} \mid z=x+i y, x>0, y \in \mathbb{R}\}
$$

and $q(z)=\phi(a, c ; z)$ is the best subordinant.

Remark 3. (a) The conclusion of this corollary has been previously obtained using different methods by Miller and Mocanu in [6] for a and $c$ real numbers satisfying the conditions:

(i) $a>0$ and $c \geq a$, or

(ii) $a \leq 0$ and $c \geq 1+\left(1+a^{2}\right)^{\frac{1}{2}}$ for $z \in U$.

(b) The result in Corollary 1 was proved in [8] by using the theory of differential subordination.

Remark 4. For $p(z)=\frac{1}{1+z}$ convex in $U, \operatorname{Re} \frac{1}{1+z}>\frac{1}{2}, z \in U$, from Theorem 3 we obtain the following corollary which gives an even more precise information on the confluent hypergeometric function's real part estimate showing how it may change by imposing other conditions on function $p$ used.

Corollary 2. Let $q(z)=\phi(a, c ; z), a, c \in \mathbb{C}, c \neq 0,-1,-2, \ldots$, defined by (6), let $\varphi: \mathbb{C}^{3} \times \bar{U} \rightarrow$ $\mathbb{C}$ and let $h$ be defined by

$$
\begin{gathered}
h(z)=\phi(a, c ; z)+z \phi^{\prime}(a, c ; z)+z^{2} \phi^{\prime \prime}(a, c ; z), z \in U . \\
\text { If } p(z)=\frac{1}{1+z}, p(0)=1, \text { a convex function in } U, \text { with } \operatorname{Re} \frac{1}{1+z}>\frac{1}{2} \text { and } \\
p(z)+z p^{\prime}(z)+z^{2} p^{\prime \prime}(z)=\frac{2 z^{2}+z+1}{(1+z)^{3}}
\end{gathered}
$$

is univalent in $U$ and satisfies

$$
\phi(a, c ; z)+z \phi^{\prime}(a, c ; z)+z^{2} \phi^{\prime \prime}(a, c ; z) \prec \frac{2 z^{2}+z+1}{(1+z)^{3}} \quad \text { or } \quad h(U) \subset \varphi(U)
$$

then

$$
\phi(a, c ; z) \prec \frac{1}{1+z}, z \in U, \quad \text { or } \quad \phi(U) \subset q(U)
$$

i.e.,

$$
\operatorname{Re} \phi(a, c ; z)>\operatorname{Re} \frac{1}{1+z}>\frac{1}{2}, z \in U
$$

or

$$
\phi(U) \subset\left\{z \in \mathbb{C} \mid z=x+i y, x>\frac{1}{2}, y \in \mathbb{R}\right\}
$$

and $q(z)=\phi(a, c ; z)$ is the best subordinant.

Proof. From relation (12) we have

$$
\phi(a, c ; z) \prec \frac{1}{1+z}, z \in U .
$$


Since $p(z)=\frac{1}{1+z}$ is a convex function in $U$, and $\operatorname{Re} \frac{1}{1+z}>\frac{1}{2}$, the differential superordination (23) is equivalent to

$$
\operatorname{Re} q(z)=\phi(a, c ; z)>\operatorname{Re} \frac{1}{1+z}>\frac{1}{2}, z \in U
$$

and $q(z)=\phi(a, c ; z)$ is the best subordinant.

Remark 5. The result in Corollary 2 was proved in [8] using the theory of differential subordination.

In the proof of the next result, Theorem 2 is invoked since first order differential superodinations for the confluent hypergeometric function are involved. The order has decreased since the method of subordination chains is applied being a well-known fact that implementing its techniques together with the theory of differential superordination has lead to easier proofs of known results and obtaining new and interesting others.

Theorem 4. Let $\phi(a, c ; z)=1+\frac{a}{c} \cdot \frac{z}{1 !}+\frac{a(a+1)}{c(c+1)} \cdot \frac{z^{2}}{2}+\ldots, a, c \in \mathbb{C}, c \neq 0,-1,-2, \ldots$, defined by (6) and let $\varphi: \mathbb{C}^{2} \rightarrow \mathbb{C}$,

$$
\varphi\left(\phi(a, c ; z), z \phi^{\prime}(a, c ; z) ; z\right)=h(z) .
$$

If $L(z, t)=\varphi\left(\phi(a, c ; z), t z \phi^{\prime}(a, c ; z) ; z\right), 0<t \leq \frac{1}{n} \leq 1, z \in U$, is a subordination chain, $p \in \mathcal{H}[1,1] \cap Q$ and $\varphi\left(p(z), z p^{\prime}(z) ; z\right)$ is univalent in $\mathcal{U}$, then

$$
h(z) \prec \varphi\left(p(z), z p^{\prime}(z) ; z\right), \quad \text { or } \quad h(U) \subset \varphi(U)
$$

implies

$$
q(z)=\phi(a, c ; z) \prec p(z), z \in U, \quad \text { or } \quad \phi(U) \subset p(U)
$$

and $q(z)=\phi(a, c ; z)$ is the best subordinant.

Proof. Since $L(z, t)$ is a subordination chain, from Definition 4 we have

$$
L(z, t) \prec L(z, 1) \text {, for } 0<t \leq 1 \text {. }
$$

For $t=1, L(z, t)$ becomes

$$
L(z, 1)=\varphi\left(\phi(a, c ; z), z \phi^{\prime}(a, c ; z)\right) .
$$

Using (27) in (24), we get

$$
L(z, 1)=h(z), z \in U
$$

Using (28), the differential superordination (26) becomes

$$
\varphi\left(\phi(a, c ; z), t z^{\prime} \phi^{\prime}(a, c ; z)\right) \prec h(z),
$$

for all $z \in U$ and $0<t \leq 1$.

The differential superordination (29) is equivalent to

$$
\varphi\left(\phi(a, c ; z), t z \phi^{\prime}(a, c ; z)\right) \in h(U) .
$$

By using Theorem 2, we have

$$
q(z)=\phi(a, c ; z) \prec p(z), z \in U .
$$


Since $q(z)=\phi(a, c ; z)$ is the univalent solution of the Equation (24), the conclusion is that $q(z)=\phi(a, c ; z)$ is the best subordinant of the differential superordination (25).

The next theorem provides means to connect the original results of this paper with the ones previously obtained in paper [8] through a sandwich-type theorem. Theorem 2 is implemented in the proof of the theorem since it contains results related to first order differential superordinations written for the confluent hypergeometric function in order the facilitate the connection with the results in paper [8].

Theorem 5. Let $h$ be convex in $U$, with $h(0)=1$, let $\gamma \in \mathbb{C} \backslash\{0\}$ with $\operatorname{Re} \gamma>0$ and consider the function $\phi(a, c ; z), a, c \in \mathbb{C}, c \neq 0,-1,-2, \ldots$ given by (6). Let $v \in \mathcal{H}[1,1] \cap Q$ and suppose that $v(z)+z v^{\prime}(z) \cdot \gamma$ is univalent in $U$.

If the differential equation

$$
h(z)=q(z)+z q^{\prime}(z) \cdot \gamma
$$

has a univalent solution $q(z)=\phi(a, c ; z)$ that satisfies $q(0)=\phi(a, c ; 0)=1$ and

$$
\phi(a, c ; z) \prec h(z), \quad \text { or } \quad \phi(U) \subset h(U)
$$

then

$$
h(z) \prec v(z)+z v^{\prime}(z) \cdot \gamma
$$

implies

$$
\phi(a, c ; z) \prec v(z), z \in U, \quad \text { or } \quad \phi(U) \subset v(U) .
$$

The function $q(z)=\phi(a, c ; z)$ is the best subordinant.

Proof. We will use Theorem 2 to prove this result.

Let $\varphi: \mathbb{C}^{2} \rightarrow \mathbb{C}$,

$$
\varphi(r, s)=r+s \cdot \gamma, r, s \in \mathbb{C}, \gamma \in \mathbb{C} \backslash\{0\}, \text { with } \operatorname{Re} \gamma>0 .
$$

Fir $r=v(z), s=z \cdot v(z)$, the relation (34) becomes

$$
\varphi\left(v(z), z v^{\prime}(z)\right)=v(z)+z v^{\prime}(z) \cdot \gamma .
$$

For $r=\phi(a, c ; z), s=z \phi^{\prime}(a, c ; z)$ and since $\phi$ is a solution of Equation (31), we have

$$
\varphi\left(\phi(a, c ; z), z \phi^{\prime}(a, c ; z)\right)=\phi(a, c ; z)+z \phi^{\prime}(a, c ; z) \cdot \gamma=h(z) .
$$

Using (35) and (36) in (33), we get

$$
\varphi\left(\phi(a, c ; z), z \phi^{\prime}(a, c ; z)\right) \prec \varphi\left(v(z), z v^{\prime}(z)\right) .
$$

For $r=\phi(a, c ; z), s=t z \phi^{\prime}(a, c ; z), 0<t \leq 1$, the relation (34) becomes

$$
\varphi\left(\phi(a, c ; z), t z \phi^{\prime}(a, c ; z)\right)=\phi(a, c ; z)+t z \phi^{\prime}(a, c ; z) \cdot \gamma .
$$

From (36), we get

$$
z \phi^{\prime}(a, c ; z) \cdot \gamma=h(z)-\phi(a, c ; z), z \in U
$$

Using (39) in (38), we have

$$
\begin{aligned}
\varphi\left(\phi(a, c ; z), t z \phi^{\prime}(a, c ; z)\right) & =\phi(a, c ; z)+t[h(z)-\phi(a, c ; z)] \\
& =\operatorname{th}(z)+(1-t) \phi(a, c ; z)
\end{aligned}
$$

$t>0,1-t \geq 0$, since $0<t \leq 1$. 
Using (32) in (40), we obtain

$$
\varphi\left(\phi(a, c ; z), t z \phi^{\prime}(a, c ; z)\right) \in h(U) .
$$

Using Theorem 2, the differential superordination (33) implies

$$
\phi(a, c ; z) \prec v(z), \quad \text { or } \quad \phi(U) \subset v(U) .
$$

Since $q(z)=\phi(a, c ; z)$ satisfies the differential Equation (31), we have that $q(z)=$ $\phi(a, c ; z)$ is the best subordinant.

If in Theorem 1 from paper [8] we take $q(z)=\phi(a, c ; z)$ and combine the result with Theorem 5, we obtain the sandwich-type theorem that follows, a form of theorems obtained when the dual theories of differential subordination and differential superordination are both used for the study on the same function, underlining the similarities and the differences between the outcomes.

Theorem 6. Let $\phi(a, c ; z)=1+\frac{a}{c} \cdot \frac{z}{1 !}+\frac{a(a+1)}{c(c+1)} \cdot \frac{z^{2}}{2 !}+\ldots, a, c \in \mathbb{C}, c \neq 0,-1,-2, \ldots$, holomorphic in $U$, given by (6), let $h$ be a convex function in $U$, let $\gamma \in \mathbb{C} \backslash\{0\}$ with $\operatorname{Re} \gamma>0$ and

$$
h(z)=\phi(a, c ; z)+z \phi^{\prime}(a, c ; z) \cdot \gamma .
$$

If $p$ and $v$ are analytic in $U$, with $p(0)=v(0)=\phi(a, c ; 0)=1, p(z)+z p^{\prime}(z) \cdot \gamma$ is analytic in $U, v(z)+z v^{\prime}(z) \cdot \gamma$ is univalent in $U$, and relation

$$
p(z)+z p^{\prime}(z) \cdot \gamma \prec \phi(a, c ; z)+z \phi^{\prime}(a, c ; z) \cdot \gamma \prec v(z)+z v^{\prime}(z) \cdot \gamma
$$

is satisfied, then

$$
p(z) \prec \phi(a, c ; z) \prec v(z), z \in U, \quad \text { or } \quad p(U) \subset \phi(U) \subset v(U) .
$$

The functions $p$ and $v$ are the best subordinant and the best dominant, respectively.

In order to conclude the study, an example is provided which shows how following the theoretical statements, interesting applications emerge.

Example 1. For $a=-1, c=i, \phi(-1, i ; z)=1+i z, z \in U$,

$$
\phi(-1, i ; 0)=1, \phi^{\prime}(-1, i ; z)=i, \phi^{\prime \prime}(-1, i ; z)=0 .
$$

Let the function $h$ be defined as

$$
\begin{aligned}
& \qquad h(z)=\phi(-1, i ; z)+z \phi^{\prime}(-1, i ; z)+z^{2} \phi^{\prime \prime}(-1, i ; z)=1+2 i z . \\
& \text { Let } p(z)=\frac{1-z}{1+z}, p(0)=1=\phi(-1, i ; 0) \text { and } \\
& \varphi\left(p(z), z p^{\prime}(z), z^{2} p^{\prime \prime}(z)\right)=p(z)+z p^{\prime}(z)+z^{2} p^{\prime \prime}(z)=\frac{-z^{3}+z^{2}+z+5}{(1+z)^{3}} .
\end{aligned}
$$

Using Corollary 1, we have

$$
\begin{array}{r}
\text { If } 1+2 i z \prec \frac{-z^{3}+z^{2}+z+5}{(1+z)^{3}} \text { or } h(U) \subset \varphi(U), \text { then } \\
1+i z \prec \frac{1-z}{1+z}, z \in U,
\end{array}
$$


or

$$
\phi(U) \subset p(U)=\{z \in \mathbb{C} \mid z=x+i y, x>0, y \in \mathbb{R}\}
$$

Indeed,

$$
\operatorname{Re}(1+i z)=\operatorname{Re}[1+i(\cos \alpha+i \sin \alpha)]=\operatorname{Re}[1-\sin \alpha+i \cos \alpha]=1-\sin \alpha>0,
$$

hence

$\phi(U)=\{z \in \mathbb{C} \mid z=x+i y, x>1-\sin \alpha, y \in \mathbb{R}\} \subset\{z \in \mathbb{C} \mid z=x+i y, x>0, y \in \mathbb{R}\}$.

\section{Discussion}

Inspired by the work of Professors Miller and Mocanu [6] and having in mind the nice results obtained by the author in [8] using the theory of differential subordination, the present paper shows the conclusions of the study on confluent (Kummer) hypergeometric function implementing the dual theory of differential superordination. The results stated in the theorems and corollaries of this paper are particularly interesting since there are not many papers published as outcomes for such an investigation. That is why the results could serve as inspiration for taking this study further. By pointing out the relation between the results presented in this paper and the previously obtained ones using other methods, it is clear that the results presented here are correct and the link between those results and the outcomes published by other authors could be investigated. Because of the sandwich theorem stated as Theorem 6, further study on the univalence of the confluent (Kummer) hypergeometric function is encouraged using the theory of differential superordination. The example provided shows that the theoretical statements can be applied and gives ideas for obtaining other interesting results using specific functions.

Funding: This research received no external funding.

Conflicts of Interest: The author declares no conflict of interest.

\section{References}

1. Miller, S.S.; Mocanu, P.T. Second order differential inequalities in the complex plane. J. Math. Anal. Appl. 1978, 65, 289-305. [CrossRef]

2. Miller, S.S.; Mocanu, P.T. Differential subordinations and univalent functions. Mich. Math. J. 1981, 28, 157-172. [CrossRef]

3. Miller, S.S.; Mocanu, P.T. Subordinants of differential superordinations. Complex Var. 2003, 48, 815-826. [CrossRef]

4. Pommerenke, C. Univalent Functions; Vandenhoeck and Ruprecht: Göttingen, Germany, 1975.

5. Branges, L. A proof of the Bieberbach conjecture. Acta Math. 1985, 154, 137-152. [CrossRef]

6. Miller, S.S.; Mocanu, P.T. Univalence of Gaussian and confluent hypergeometric functions. Proc. Am. Math. Soc. 1990, 110, 333-342. [CrossRef]

7. Miller, S.S.; Mocanu, P.T. Differential Subordinations. Theory and Applications; Marcel Dekker, Inc.: New York, NY, USA; Basel, Switzerland, 2000.

8. Oros, G.I. New Conditions for Univalence of Confluent Hypergeometric Function. Symmetry 2021, 13, 82. [CrossRef]

9. Magesh, N.; Jothibasu, J.; Murthy, S. Subordination and superordination results associated with the generalized hypergeometric function. Math. Slovaca 2014, 5, 1197-1216. [CrossRef]

10. Murugusundaramoorthy, G.; Magesh, N.; Raina, R.K. Subordination and superordination properties for analytic functions involving Wright's functions. Le Mat. 2011, 66, 65-77.

11. Cho, N.E.; Aouf, M.K.; Srivastava, R. The principle of differential subordination and its application to analytic and p-valent functions defined by a generalized fractional differintegral operator. Symmetry 2019, 11, 1083. [CrossRef] 